\title{
Minimally-Invasive Parathyroid Surgery
}

\author{
David Rosen, Joseph Sciarrino and Edmund A. Pribitkin \\ Thomas Jefferson University, Philadelphia, PA
}

USA

\section{Introduction}

Parathyroid surgery was first performed to correct primary hyperparathyroidism less than 100 years ago, and surgical treatment remains the only successful and durable cure for the disorder. 1,2 Techniques have evolved over the past century and continue to change and develop to this day. The conventional technique of bilateral neck exploration, though effective, has the disadvantage of being an invasive procedure, resulting in greater pain, poorer cosmesis, longer operative time, and longer hospitalization. More recently, developments in adjunctive technologies have allowed the development of less invasive techniques to achieve the same end result. This chapter will briefly discuss the conventional surgical treatment of primary hyperparathyroidism followed by a look at the minimally invasive techniques that are being developed and used today.

\section{Anatomy and embryology}

Knowledge of the anatomy and embryology of the parathyroid glands is paramount to the success of surgery, regardless of the techniques employed. The variability in gland position can make localization difficult both pre-operatively and intra-operatively. The parathyroids are endocrine glands that develop from the endoderm of the $3^{\text {rd }}$ and $4^{\text {th }}$ pharyngeal pouches beginning in the $5^{\text {th }}$ week of gestation. They migrate from this position inferiorly, reaching their final locations by the $7^{\text {th }}$ week. The $3^{\text {rd }}$ pharyngeal pouch develops into both the thymus and the inferior parathyroids, while the $4^{\text {th }}$ arch becomes the superior glands.1-3 Parathyroid glands are usually about $5 \times 3 \times 1 \mathrm{~mm}$ in size with an average weight of $35 \mathrm{mg}$, although adenomatous glands may be much larger. ${ }^{3}$

Normally, each set of glands is paired, resulting in 2 superior and 2 inferior glands. This is the case in $84 \%$ of patients. About $3 \%$ of patients will have only 3 glands, and $13 \%$ of patients may have 5 or more glands. The superior parathyroid glands normally reside postero-medial to the superior thyroid lobes, near the cricothyroid junction, while the inferior glands tend to be on the postero-lateral side of the inferior thyroid lobe, inferior to where the recurrent laryngeal nerve and inferior thyroid artery cross. The inferior parathyroids are usually found within $2 \mathrm{~cm}$ of the lower pole of the thyroid. This anatomic arrangement of glands is true in about $80 \%$ of patients. However, aberrant migration is common, and the glands can be found in ectopic locations in many cases. Ectopic superior parathyroid glands may be retroesophageal, intrathyroidal, or in the posterior mediastinum. Inferior parathyroid glands have more variable ectopic sites as a 
result of their longer migration. These sites include the thyrothymic tissue, thyroid, thymus, anterior mediastinum, and within the carotid sheath. Understanding of this anatomic variability is important in interpreting preoperative imaging and directing operative exploration..$^{1-3}$

\section{Pathophysiology}

Overproduction of parathyroid hormone $(\mathrm{PTH})$ is the defining feature of hyperparathyroidism. Hyperparathyroidism may be caused by a single parathyroid adenoma, multi-gland hyperplasia, double adenomas, or parathyroid carcinoma. Single adenoma accounts for about $81-96 \%$ of hyperparathyroidism, depending on the series. Multigland hyperplasia accounts for $4-14 \%$, double adenomas $2-11 \%$, and parathyroid carcinoma $<1 \% .1,2,4$ Traditional bilateral neck exploration remains the standard of care for parathyroid carcinoma ${ }^{1}$ so the focus of this chapter on minimally invasive techniques will be on benign disease.

\begin{tabular}{|c|c|}
\hline Single adenoma & $81-96 \%$ \\
\hline Multi gland hyperplasia & $4-14 \%$ \\
\hline Double adenoma & $2-11 \%$ \\
\hline Carcinoma & $<1 \%$ \\
\hline
\end{tabular}

Table 1. Causes of Primary Hyperparathyroidism

\section{Traditional bilateral neck exploration}

Traditional bilateral neck exploration (BNE) was the primary approach used by parathyroid surgeons until this past decade when minimally invasive techniques became more prevalent. Even as recently as 1998 nearly $3 / 4$ of parathyroid surgeons were still performing bilateral cervical exploration as the primary surgical technique. ${ }^{3}$ In the absence of other significant contraindications, all patients with diagnosed primary hyperparathyroidism, tertiary hyperparathyroidism, or select cases of secondary hyperparathyroidisim are candidates for this operation, and no preoperative localization studies are required, as all glands should be visualized intraoperatively. 1,2,5

BNE requires general anesthesia, typically with endotracheal intubation and often with a nerve integrity monitoring tube. A midline, transverse 3-5 cm cervical incision is made and carried through platysma, subplatysmal flaps elevated, the strap muscles divided in midline, and dissection and exploration continued until all parathyroid glands are visualized. This typically entails anteromedial retraction of the thyroid lobe to reveal the parathyroids posteriorly. Care is taken to safeguard the recurrent laryngeal nerve. Glands that are abnormal in appearance are resected. Those that are questionable may be biopsied and sent for frozen section pathology to guide decision of whether or not to resect. If all glands appear abnormal as is the case with multi-gland hyperplasia, the surgeon may perform a subtotal or total resection with autotransplantation. A subtotal resection, also 
known as a 3.5 gland resection is accomplished by resecting 3 abnormal glands, and approximately $1 / 2$ of the most normal-appearing gland, leaving approximately $50 \mathrm{mg}$ of parathyroid tissue. This approach removes the majority, but not all of the PTH secreting tissue in an attempt to allow the patient to become normocalcemic without needing chronic vitamin D and calcium supplementation. A total resection with autotransplantation involves resecting all found glands and then implanting small sections of the gland into a distant site; typically 12-24 sites within the subcutaneous tissue or the brachioradialis of the nondominant forearm. Again, this maintains functioning parathyroid tissue to reduce the risk of lifetime supplementation and permits titration of hormone level by future selective removal of auto-transplanted parathyroid tissue. $1,2,5$

Intraoperative PTH monitoring is frequently used to determine completeness of resection of abnormal tissue. If PTH does not drop to $<50 \%$ of the pre-operative level, then the exploration is continued in the neck and mediastinum to look for ectopic tissue. If all ectopic sites have been explored and the Miami criterion has not been met, the operation is concluded and further work-up postoperatively with imaging must be done.1-3,6,7

Cure rates have been reported at $>95 \%$ with a single operation, and complications at $<4 \%$. 1,2,5 Complications include recurrent laryngeal nerve (RLN) paresis, persistent hypocalcemia, and hematoma. Postoperatively patients are admitted at least overnight, and postoperative calcium levels are followed to screen for hypocalcemia. Patients are typically placed on vitamin D and calcium postoperatively and go home on this supplementation. ${ }^{1}$

Despite the decrease in its incidence (10\% of endocrine surgeons surveyed in 2008 used BNE as their primary technique, as opposed to $74 \%$ in $1998^{3}$ ), bilateral exploration is still the preferred primary technique in cases of MEN, non-MEN familial isolated hyperparathyroidism (both of which carry an increased risk of multi-gland disease), and non-localizing pre-op imaging. ${ }^{2}$ Additionally, a minimally invasive technique failure may need to be converted to a bilateral neck exploration to achieve a cure.

\section{Minimally invasive techniques}

The term "minimally invasive" is applied to several different techniques. These techniques share the objectives of reduced dissection, operative time, and duration of hospitalization as well as an improvements in patient comfort and cosmesis through smaller or more discretely located incisions. Technological advancements in imaging, laboratory, and operative techniques have made these approaches possible. These adjuncts will be discussed, followed by an explanation of the techniques themselves.

\section{Preoperative localization studies}

\subsection{Parathyroid scintigraphy}

This imaging technique uses a radiotracer (usually $99 \mathrm{mTc}$-sestamibi) injected intravenously to locate the parathyroid glands. ${ }^{99 m T c-s e s t a m i b i ~ p r e f e r e n t i a l l y ~ d i s t r i b u t e s ~ t o ~ c e l l s ~ w i t h ~ h i g h ~}$ concentrations of mitochondria, resulting in greater concentration in cells of thyroid, heart, liver, salivary gland, and parathyroid tissue. Parathyroid glands that are hyperplastic or adenomatous tend to concentrate sestamibi to levels significant for detection, while normal 
glands are not typically seen. This is likely due to increased mitochondria as well as increased blood flow to these glands. Due to their anatomic relationship and shared affinity for the radiotracer, signal from the thyroid and parathyroids may overlap, obscuring the definition of an abnormal parathyroid gland. Fortunately, the retention of the radiotracer over time is greater in parathyroid than thyroid tissue. Combining early and delayed (2-3 hours) imaging permits better identification of abnormal parathyroid tissue. Additionally, an abnormal signal contour or a signal clearly separate from the thyroid bed raises suspicion of abnormal parathyroid tissue on either early or late images.3,8 Sestamibi scanning may be done with planar imaging, or with 3-dimensional imaging using singlephoton emission computed tomography (SPECT). SPECT has been reported to allow better differentiation of parathyroid tissue from the thyroid gland, and thus better detection and localization. ${ }^{1-3}$

This technology does have limitations, however. Uptake and retention of the radiotracer by abnormal parathyroid tissue may be variable. If washout from an adenoma is rapid, no discrete signal will be seen on the delayed images, despite the presence of a diseased gland (false negative). False negatives are also more commonly seen in patients with multi-gland disease, such as double adenomas or multi-gland hyperplasia. Additionally, multiple factors may cause false positive results, such as adenomas of thyroid origin, lymph nodes, or multinodular goiter, all of which have affinity for $99 \mathrm{mTc}$-sestamibi and can be located in the same region as an abnormal gland.2,3Arguably, the greatest utility of sestamibi scanning is in the identification of ectopically located parathyroid tissue.

\subsection{Ultrasonography}

This relatively low-cost modality has the advantages of the absence of radiation and providing anatomic information in the area of intended surgery. Moreover, ultrasonography enables excellent visualization of the thyroid gland and can diagnose concurrent thyroid disease, limiting re-operation rates. Normal parathyroid glands are typically not seen on ultrasound due to their small size and location. Adenomatous glands tend to appear homogenous on ultrasound and are usually hypoechoic to the thyroid gland signal. The use of Doppler imaging can provide information regarding parathyroid galnd vascularity and can identify an artery feeding an adenomatous gland, which greatly increases the accuracy of diagnosis. ${ }^{3}$

Shortcomings of ultrasound include poor sensitivity for some ectopic glands such as retrotracheal or mediastinal glands. Glands in these locations are shadowed by the tracheal air column and bones of the sternum and clavicle, respectively. Large adenomas may also complicate diagnosis, because their imaging characteristics may be atypical. They can appear heterogeneous and/or hyperechoic to thyroid tissue. Disease of the thyroid such as mulitinodular goiter or posterior thyroid nodules may also increase the difficulty of detection of parathyroid adenomas. Enlarged lymph nodes associated with anthracotic pigment, thyroiditis and malignant thyroid disease can also confound parathyroid localization. However, as previously mentioned, even a study that fails to reveal a parathyroid adenoma may be useful by identifying thyroid disease in a patient that is being considered for surgery for hyperparathyroidism. Incidence of concurrent thyroid disease has been reported as high as $51 \%$ in patients being considered for parathyroid surgery, and the incidence of thyroid malignancy as $2-6 \% .2,3$ 


\subsection{Combined scintigraphy and ultrasonography}

Radiotracer imaging and ultrasound alone show similar sensitivities. Sensitivity for sestamibi scanning has been reported in the range of $68-95 \%$ for single adenomas, with one meta-analysis putting it at $88 \%$. Sensitivity is far less for multi-gland disease and has been reported at $44 \%$ for hyperplasia and $30 \%$ for double parathyroid adenomas. ${ }^{1-4,6,8,10}$ Sensitivity of ultrasound alone has been reported at $72-89 \%$ for patients with single adenomas. Again, sensitivity drops for multi-gland disease and has been reported at $16 \%$ for parathyroid hyperplasia and $35 \%$ in double adenomas. $1-4,6,8,10$

Ultrasonography and Radio-guided imaging complement each other and increase diagnostic accuracy when used together. The surgeon may use both the functional information from scintigraphy along with the anatomic information from ultrasound. Additionally, ectopic glands that are missed by ultrasonography may be detected with scintigraphy, while ultrasonography may detect thyroid abnormalities helping to interpret scintigraphy findings. Combining these techniques results in a sensitivity of 74$95 \%$ for single gland disease. However, the sensitivity for double adenomas is $60 \%$, and multi-gland disease is only accurately predicted $30 \%$ of the time by these techniques. When the two imaging studies are concordant (which occurs in 50-60\% of cases) the sensitivity is in the range of $94-99 \% .1,3$ In fact, some surgeons suggest that intraoperative PTH monitoring not be used in cases of concordant ultrasound and sestamibi scan, and simply terminate the procedure after excising the gland indicated on the imaging studies. Combined ultrasonography and sestamibi is the preferred imaging method of most parathyroid surgeons. ${ }^{1}$

\subsection{Other imaging techniques}

Computed tomography [CT] and magnetic resonance imaging [MRI] scans may provide additional anatomical information, but are not first-line studies. CT offers the advantage of scanning the entire neck and mediastinum to help with localization of ectopic glands. Sensitivity of CT scanning alone ranges from $46-87 \%$. When combined with ultrasonography, it results in only slightly increased sensitivity compared with ultrasonography alone. CT tends to be used only in patients undergoing reoperation or in patients with an ectopic gland detected on sestamibi.1,3-5,8

CT can also be combined with SPECT to give images that contain a combination of anatomic and functional information. This allows better localization and sensitivities ranging from 88$93 \%$. Benefits may be greater in the subset of patients with multi-gland disease or goiter, but such combination scans require further investigation. ${ }^{3}$

MRI has sensitivities rivaling other modalities, but its high cost and other options for imaging have limited its use to select cases. $1,3-5,8$

\section{Introperative adjunctive techniques}

\subsection{Intraoperative parathyroid hormone monitoring}

The serum half-life of parathyroid hormone (PTH) is 3-5 minutes. This short turn-over time along with the availability of rapid assays that take from 8-20 minutes for results allow the 
operative team to monitor and predict the success of surgery based on the amount of circulating PTH. Typically, pre-operative and pre-incision blood samples are taken for baseline measurements. After excision of all of the suspected diseased tissue, samples are usually sent at 0,5 , and 10 minutes. The criteria used for a successful operation at most centers is a drop of the PTH level to $50 \%$ or less of the pre-incision level. If this occurs at 5 or 10 minutes, the operation is deemed complete. If it does not occur, further cervical exploration is performed to identify additional parathyroid tissue that may be causing the patient's hyperparathyroidism, and the suspicion for multi-gland disease increases. If the $50 \%$ criterion is met, surgical success rates (as measured by postoperative normocalcemia) are in the range of $97-98 \%$. This technique may be used in both minimally invasive and bilateral neck exploration techniques. Additionally, rapid PTH assay may be performed on excised tissue, allowing rapid identification of parathyroid tissue if there is any doubt.1,2,4-7

\subsection{Gamma probe}

The radioactivity of $99 \mathrm{~m}$ Tc-sestamibi may also be detected by a hand-held probe. This adjunctive technology can help direct dissection as well as provide information regarding completeness of excision. This will be further discussed in the section regarding radioguided parathyroid surgery.

\section{Minimally invasive operative techniques}

\subsection{Focused parathyroidectomy}

Focused parathyroid surgery is possible and effective because of the availability of preoperative localization studies and the fact that about $75-90 \%$ of hyperparathyroidism is due to single adenomas. These factors allow for limited dissection in the area of the diseased gland, decreasing the invasiveness of bilateral neck dissection.

Candidates for this surgery are patients who meet the guidelines for surgical treatment of hyperparathyroidism, have positive pre-operative localization imaging, and do not have a condition that would predispose them to multi-gland disease, such as MEN or non-MEN familial isolated hyperparathyroidism. Patients with such conditions or non-localizing preoperative imaging should undergo traditional or minimally invasive bilateral neck exploration instead. ${ }^{1,2}$

This technique involves making a small $(2-4 \mathrm{~cm})$ transverse incision in a skin crease in the midline or on the side indicated by pre-operative imaging. The incision is carried down to the strap muscles, which are dissected and lateralized. The thyroid is mobilized and retracted medially for access. Directed dissection based on preoperative imaging allows identification of the offending gland, while care is taken not to injure the recurrent laryngeal nerve. Since the other parathyroid glands are not visualized in this technique, intraoperative parathyroid hormone monitoring is employed to determine completeness of the operation. If the $50 \%$ criterion is not met, explorative dissection to visualize the other glands is employed.1,2,5,6,11

Given the limited amount of dissection, this procedure may be performed under sedation with local and regional anesthesia instead of general anesthesia.1,2 This helps reduce the risk of anesthesia in these cases. Whether general or local anesthesia is used, the majority of 
cases can be performed on an outpatient basis. Postoperative calcium levels do not typically have to be checked because the remaining parathyroid glands remain undisturbed. For this same reason, patients do not typically require postoperative supplementation with calcium and vitamin D. Hungry bone syndrome may still occur, however, and inpatient hospitalization and calcium testing should be performed when the concern of postoperative hypocalcemia is high.1,2,5

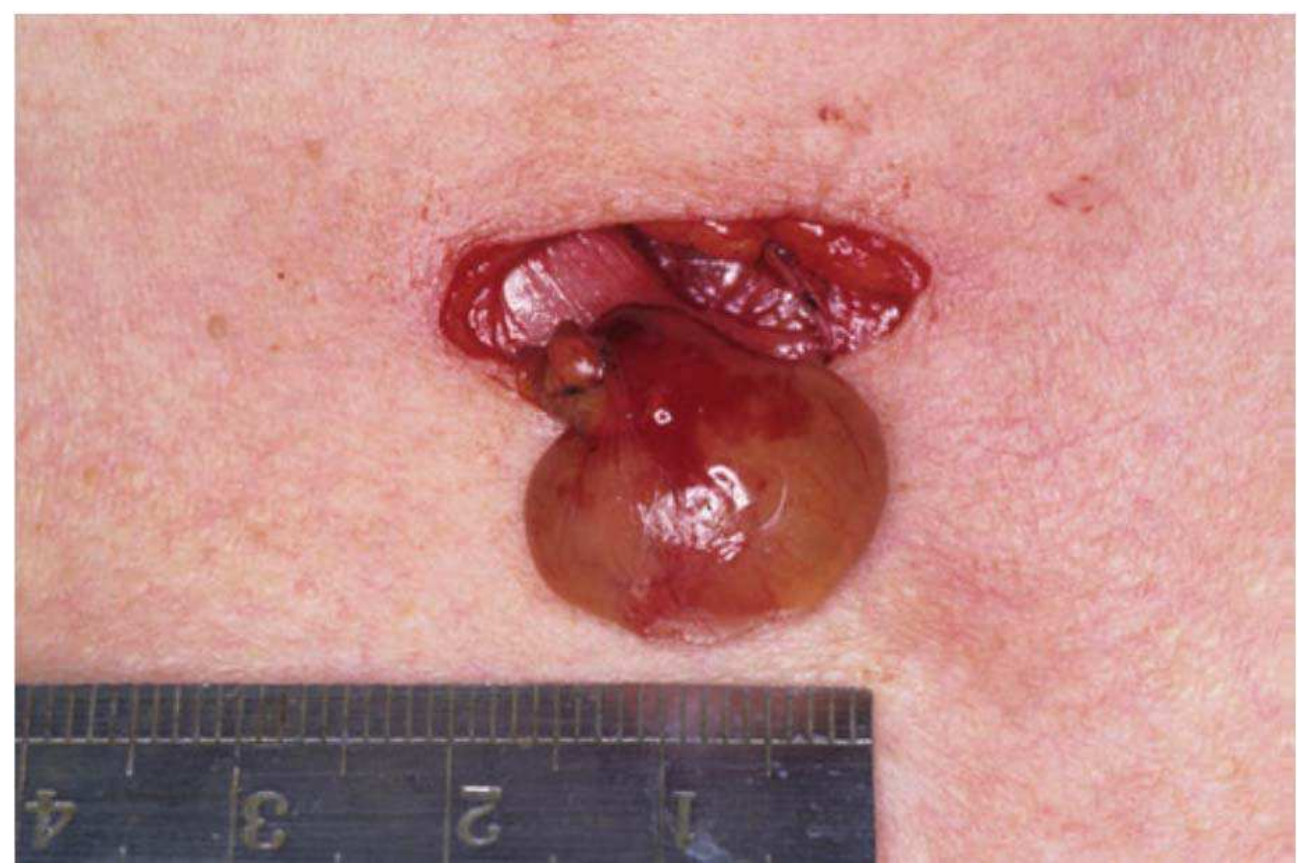

Fig. 1. An example of a $2 \mathrm{~cm}$ incision possible with minimally invasive techniques.

$$
\begin{array}{ll}
\text { - } & \text { Failure of pre-operative localization } \\
\text { - } & \text { Known multigland disease } \\
\text { - } & \text { MEN } \\
\text { - } & \text { Lithium-MEN familial isolated hyperparathyroidism } \\
\text { - } & \text { Parathyroid carcinoma }
\end{array}
$$

Table 2. Contraindications to Focused Exploration Techniques for Parathyroidectomy

Benefits of this approach over bilateral neck exploration include shorter operative time, shorter hospitalization, and the avoidance of general anesthesia.1,2,5 These factors contribute to a decreased overall cost of the procedure. One surgeon compared 613 BNE operations to 1037 focused parathyroidectomies under local and regional anesthesia, and found the average cost savings in his institution to be $\$ 1471$ per case. ${ }^{5}$ Additionally, cosmesis is typically improved with this technique due to a smaller incision. ${ }^{1,2,5,11}$ One disadvantage of 
this technique is the failure to visualize all parathyroids resulting in the potential risk of missing an abnormal gland which may occur in $10-25 \%$ of cases. However, the use of IPM helps to decrease this risk.

Outcomes using this technique are generally comparable to the traditional approach, with relatively high cure (95-99\%) and low complication (1-4\%) rates. Many studies have confirmed that this is a viable alternative to the traditional bilateral neck exploration. $1,2,5,11$

\subsection{Radio-guided surgery}

99mTc-sestamibi radioactivity can be detected by a handheld gamma probe which can be used intraoperatively to help locate the hyperfunctioning parathyroid tissue. This technique involves intravenous injection of the radiotracer 2 hours prior to surgery. In the operating room, the gamma probe can be used to determine on which side of the neck to place the incision. As in focused parathyroidectomy, efforts are made to keep the incision as short as possible $(2-4 \mathrm{~cm})$ without limiting the exposure. After the skin and platysma are incised, the probe can be inserted into the wound and the dissection directed in the area of highest radioactivity. Dissection is continued until the hyperfunctioning parathyroid gland is encountered and excised.2,4,8 The excised tissue can be placed directly against the probe to measure its radioactivity relative to a background level that is found by placing the probe over the thyroid isthmus. If the ex-vivo count of the excised tissue is $>20 \%$ of the background radiation at the thyroid isthmus, it is strong evidence that the excised tissue is indeed parathyroid adenoma. Hyperplastic parathyroid glands tend to exhibit $<16 \%$ of background radiation, and normal parathyroids, fat, and lymph nodes are usually around $2 \% .4,8$ Much like the focused parathyroidectomy technique previously described, this technique benefits from the ability to use local and regional anesthesia with sedation. Also, it too is often used in conjunction with IPM.

Like the focused parathyroidectomy technique, this approach benefits from a small incision, limited dissection, and potential for avoidance of general anesthesia and inpatient hospitalization. Use of the gamma probe was found to have a $93-94 \%$ sensitivity and $88 \%$ positive predictive value in localization of a parathyroid adenoma. $2,4,8$ However, failure of localization does occur, and conversion rates to convential bilateral exploration have been found to be $10 \%$ inpatients with single adenomas, and $50 \%$ for multi-gland disease and hyperplasia, though similar rates have been observed in focused parathyroidectomy. 2,5 Additionally, logistical concerns over timing of surgery and equipment may be seen as a disadvantage of this technique.

Radio-guided minimally invasive bilateral neck exploration has been advocated by high volume thyroid centers as yielding optimal cure rates while decreasing costs. Norman et al have noted that even highly selected unilateral explorations in patients with a clearly positive, "in focus" sestamibi scan with a solitary localization of radioactivity clearly distinct from a normal thyroid gland can still fail to achieve cure in up to $6 \%$ of cases. Following a planar sestamibi scan performed two hours before surgery, through a $2.5 \mathrm{~cm}$ incision under general laryngeal masked anesthesia, Norman identifies each of the four parathyroid glands and determines its metabolic activity by removing a portion of the gland and measuring the contained gamma radioactivity against a standard curve of hormone production. This permits classification of each gland as normal (dormant), adenoma, hyperplastic or clinically 
enlarged non-dormant. By protocol, all glands that are non-dormant are removed with more than 1 gland removed in $24.7 \%$ of cases. ${ }^{12}$ Norman employs neither frozen section analysis nor intra-operative PTH assay, and operative times average 22.3 minutes per case. One, three and ten year cure rates for radioguided minimally invasive bilateral neck exploration exceed $99 \%$ in this case series.

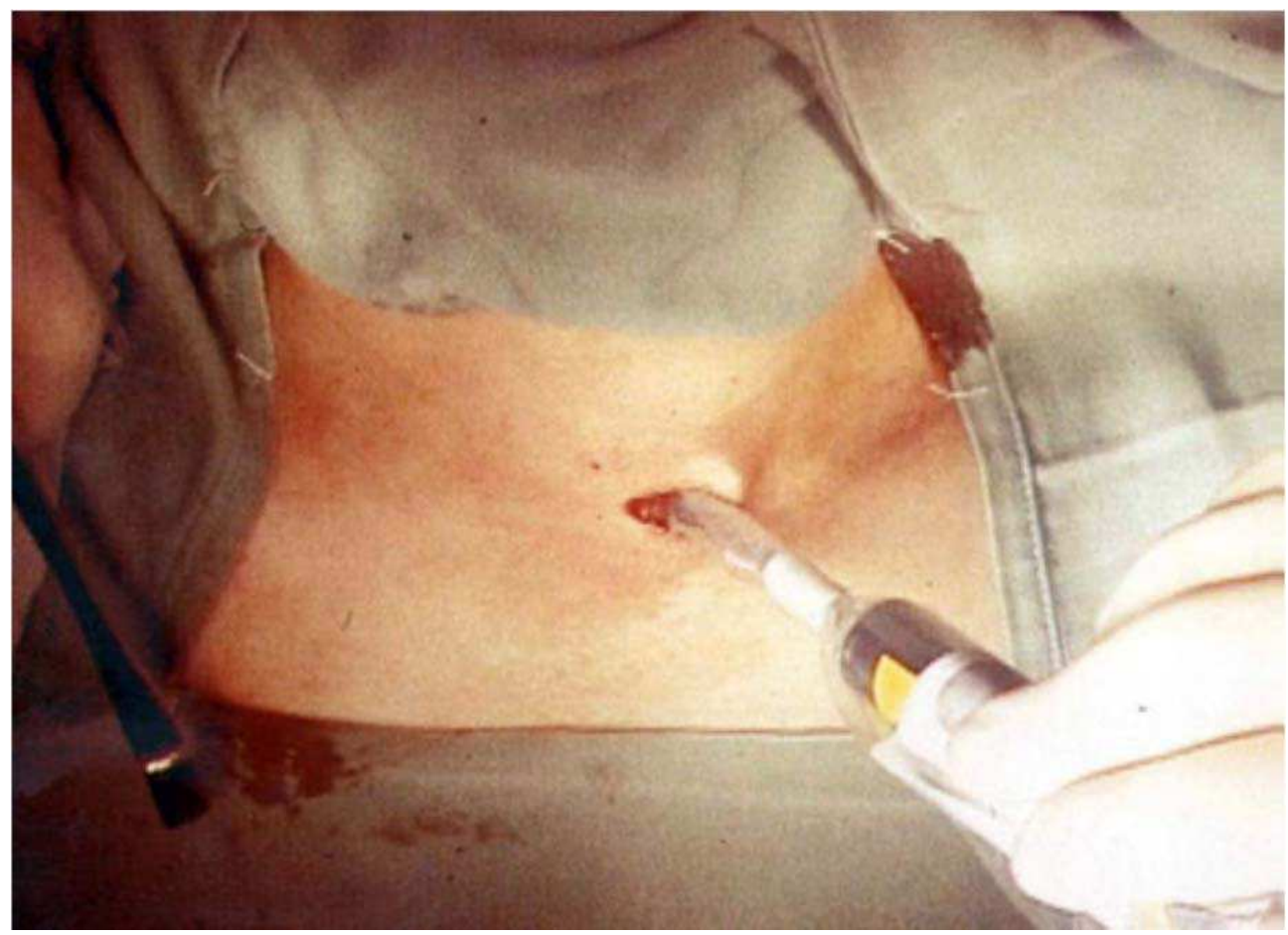

Fig. 2. A gamma probe inserted into the incision to guide dissection.

\subsection{Endoscopic parathyroidectomy}

Endoscopic parathyroidectomy attempts to take the techniques developed for minimally invasive laparoscopic surgeries and apply them to the neck. This technique was first reported by Gagner in 1996 for a bilateral cervical exploration..$^{13}$ It uses several very small incisions (about $5 \mathrm{~mm}$ ) as ports for an endoscope and endoscopic instruments. Generally, a $5 \mathrm{~mm}$ trocar is inserted superior to the sternal notch in the midline of the neck through which a $30^{\circ}$ endoscope is placed. Three additional ports are placed, two in the right neck and one in the left. Working space is created by insufflation of $\mathrm{CO}_{2}$. The strap muscles are divided at the raphe, and the thyroid is mobilized antero-medially to reveal the parathyroid glands for resection.1,2,9,13 Other techniques include approaches from the lateral neck, anterior chest wall, and axilla. Some of these techniques change the trocar sites to improve cosmesis. Others, such as the lateral approach, attempt to improve access to superior glands - although the technique described by Gagner provides excellent access to the lower pole of the thyroid, access to the superior poles is limited. ${ }^{2}$ 
These endoscopic approaches provide the benefit of improved cosmesis by reducing the incisions on the neck to small port sites, which in some cases are covered by clothing. Additionally, focused parathyroidectomy and bilateral cervical exploration both may be carried out with this technique. One important advantage of this method is the ability to visualize and dissect in the mediastinum if ectopic glands are suspected. Some have argued that the magnification of the endoscope allows better visualization of the recurrent laryngeal nerve, while others state that visualization of the never is poorer due to less exposure.1,2,9,13

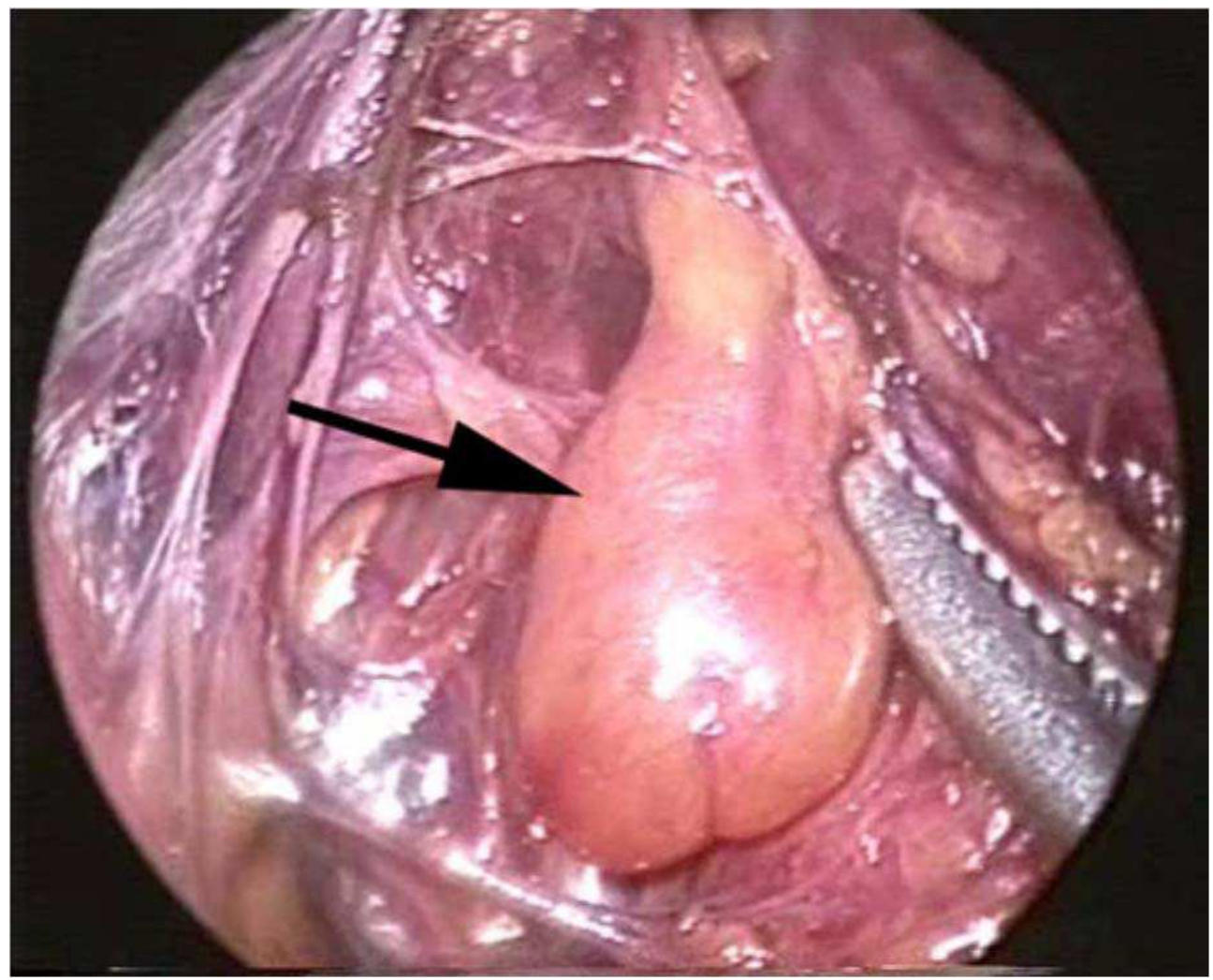

Fig. 3. View of a parathyroid adenoma through an endoscope.

A major disadvantage of this method is the steep learning curve for the surgical team to become proficient with the technique. Dedicated equipment must be purchased and maintained. Most endoscopic approaches to parathyroid surgery tend to have longer overall operative time, particularly in the early part of the learning curve. Some complications specific to this technique include subcutaneous emphysema, hypercapnia, respiratory acidosis, tachycardia, and air embolism.1,2,9,13 These may be reduced by lower-pressure insufflation used in some approaches. ${ }^{9}$ Also, the surgeon loses the tactile assessment that is possible in an open approach, and violation of the parathyroid capsule may be more likely when removing an adenoma from a small port. ${ }^{2}$ Despite these disadvantages, cure rates with this technique are comparable to the previous techniques listed.1,2,9 


\subsection{Minimally invasive video-assisted parathyroidectomy}

Minimally Invasive Video-Assisted Parathyroidectomy (MIVAP) was first described by Miccoli in 1998. ${ }^{14}$ It is considered a gasless endoscopic technique, and, as with focused parathyroidecotmy, relies on preoperative localization studies and IPM. Like the focused technique, MIVAP is not an option for patients with multi-gland disease, conditions predisposing to multi-gland disease, parathyroid carcinoma, or failed preoperative localization. Additionally, patients with large goiters are not candidates for this approach.2,15

A $15-20 \mathrm{~mm}$ incision is made in the midline, and the strap muscles are divided in at the raphe. Blunt dissection on the side of the neck as indicated by localization studies, and the strap muscles are retracted laterally from the thyroid using direct visualization. Then, a $5 \mathrm{~mm} 30^{\circ}$ endoscope is inserted into the wound. Working space is created by use of external retractors, so insufflation of $\mathrm{CO}_{2}$ is not necessary. Specialized $2 \mathrm{~mm}$ endoscopic instruments are used to complete the dissection of the parathyroid adenoma and excise it from the surrounding tissue. If the adenoma is not found, or if PTH levels do not drop appropriately, the procedure is converted to a conventional bilateral neck exploration.1,2, 14, 15

The absence of insufflation in this technique avoids many of the complications of the total endoscopic approach. Also, operative times tend to be shorter than total endoscopic procedures. Miccoli reported an average operative time of 36.2 minutes in a series of 370 operations, and an average time of 25.7 minutes for the last 100 in that series. ${ }^{15}$ This procedure also affords the surgeon tactile assessment of the surgical bed, which is not available with total endoscopic approaches. However, the disadvantages of a long learning curve and specialized equipment remain. Also, 2 assistants are required for this technique.1,2

Cure rates with MIVAP are comparable to the other procedures described and have been reported at $96-100 \%$. Complication rates are also comparable to the other approaches.1,2,15

\section{Conclusion}

Although first performed nearly 100 years ago, parathyroid surgery has undergone rapid evolution over the past few decades. Advances in imaging, laboratory assay, and operative technique have made new methods possible. The varied minimally invasive techniques described in this chapter are all capable of producing satisfactory outcomes, and many offer significant advantages over traditional bilateral cervical exploration. Nonetheless, cure rates approaching $100 \%$ can only be achieved through evaluation of all four glands ${ }^{16}$. Parathyroid surgeons must be well versed in both traditional and minimally invasive techniques. As cure rates are high among all techniques listed, future refinement and innovation are likely to be directed at reducing complications, lowering overall cost, and improving patient satisfaction.

\section{References}

[1] M. Augustine, P. Bravo, M. Zeiger. Surgical Treatment of Primary Hyperparathyroidism. Endocrine Practice. Volume 17. Supplement 1 / March-April 2011.

[2] John I. Lew, Carmen C. Solorzano. Surgical Management of Primary Hyperparathyroidism: State of the Art. Surgical Clinics of North America. Volume 89. Issue 5. October 2009: Pages 1205-1225. 
[3] N. Johnson, S. Carty, M. Tublin. Parathyroid Imaging. Radiol Clin N Am. Volume 49. Issue 3. May 2011: 489-509.

[4] H. Chen, E. Mack, J.R. Sterling. A Comprehensive Evaluation of Perioperative Adjuncts During Minimally Invasive Parathyroidectomy. Ann Surg September2005: 242(3): 375-383.

[5] R. Udelsman, Z. Lin, P. Donovan. The Superiority of Minimally Invasive Parathyroidectomy Based on 1650 Consecutive Patients With Primary Hyperparathyroidism. Ann Surg. Volume 253. Issue 3. March 2011: 585-591.

[6] H. Takami, Y. Ikeda, N. Wada Surgical management of primary hyperparathyroidixsm. Biomedicine \& Pharmacotherapy. Volume 54. Supplement 1. June 2000: Pages 17s-20s

[7] D. Canerio-Pla. Contemporary and Practical Uses of Intraoperative Parathryoid Hormone Monitoring. Endocrine Practice. Vol 17. Suppl 1. March/April 2011: 44-53.

[8] Y. Ikeda, J. Takayama, H. Takami. Minimally Invasive Radioguided Parathyroidectomy for Hyperparathyroidism. Annals of Nuclear Medicine. Volume 24. Number 4. March 2010: 233-240.

[9] Y. Ikeda, H. Takami, G. Tajima, Y. Sasaki, J. Takayama, H. Kurihara, M. Niimi. Section 1. Parathyroid: Total Endoscopic Parathyroidectomy. Biomed Pharmacotherapy. Volume 56. Supplement 1. 2002: 22s-25s.

[10] M. Weiss, R. Schmid, M. Hacker, T. Pfluger. Hyperparathryoidism: How to Optimize Parathyroid Imaging by Means of Tc-99m Sesta-MIBI Scintigraphy and Ultrasound? The Endocrinologist. Volume 17. Number 1. February 2007: 50-56.

[11] Y. Ikeda, H. Takami, G. Tajima, Y, Sasaki, J. Takayama, H. Kurihara, M. Niimi. Section 1: Parathyroid: Direct Mini-Incision Parathyroidectomy. Biomed Pharmacotherapy. Volume 56. Suplement 1. 2002: 14s-17s.

[12] Norman J, Politz D. Measuring individual parathyroid gland hormone production in real time during radio guided parathyroidectomy. Experience in over 8,000 operations. Minerva Endocrinology. Volume 33, Issue 3. September 2008: 147-157.

[13] M. Gagner. Endoscopic subtotal parathyroidectomy in patients with primary hyperparathyroidism. Br J Surg. 83. 1996: 875 [letter].

[14] P. Micoli, C. Bendinelli, E. Vignali, S. Mazzeo, G. Matteo Cecchini, L. Pinchera, C. Marcocci. Endoscopic Parathyroidectomy: Report of an Initial Experience. Surgery. Volume 124. Issue 6. December 1998: 1077-1080.

[15] P. Miccoli, P. Berti, G. Materazzi, M. Massi, A. Picone, M. Minuto. Results of Videoassisted Parathyroidectomy: Single Institution's Six-year Expierence. World J Surg. Volume 28. Number 12. December 2004: 1216-1218.

[16] Norman J. Controversies in Parathyroid Sugery: The Quest of a "mini" unilateral operation seems to have gone too far. J Surg Oncol. 2011. In press. 


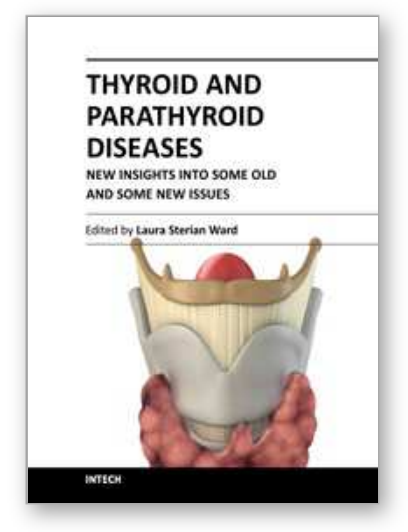

\section{Thyroid and Parathyroid Diseases - New Insights into Some Old and Some New Issues}

Edited by Dr. Laura Ward

ISBN 978-953-51-0221-2

Hard cover, 318 pages

Publisher InTech

Published online 07, March, 2012

Published in print edition March, 2012

This book was designed to meet the requirements of all who wish to acquire profound knowledge of basic, clinical, psychiatric and laboratory concepts as well as surgical techniques regarding thyroid and parathyroid glands. It was divided into three main sections: 1. Evaluating the Thyroid Gland and its Diseases includes basic and clinical information on the most novel and quivering issues in the area. 2. Psychiatric Disturbances Associated to Thyroid Diseases addresses common psychiatric disturbances commonly encountered in the clinical practice. 3. Treatment of Thyroid and Parathyroid Diseases discusses the management of thyroid and parathyroid diseases including new technologies.

\section{How to reference}

In order to correctly reference this scholarly work, feel free to copy and paste the following:

David Rosen, Joseph Sciarrino and Edmund A. Pribitkin (2012). Minimally-Invasive Parathyroid Surgery, Thyroid and Parathyroid Diseases - New Insights into Some Old and Some New Issues, Dr. Laura Ward (Ed.), ISBN: 978-953-51-0221-2, InTech, Available from: http://www.intechopen.com/books/thyroid-and-parathyroiddiseases-new-insights-into-some-old-and-some-new-issues/minimally-invasive-parathyroid-surgery

\section{INTECH}

open science | open minds

\section{InTech Europe}

University Campus STeP Ri

Slavka Krautzeka 83/A

51000 Rijeka, Croatia

Phone: +385 (51) 770447

Fax: +385 (51) 686166

www.intechopen.com

\section{InTech China}

Unit 405, Office Block, Hotel Equatorial Shanghai

No.65, Yan An Road (West), Shanghai, 200040, China

中国上海市延安西路65号上海国际贵都大饭店办公楼405单元

Phone: +86-21-62489820

Fax: $+86-21-62489821$ 
(C) 2012 The Author(s). Licensee IntechOpen. This is an open access article distributed under the terms of the Creative Commons Attribution 3.0 License, which permits unrestricted use, distribution, and reproduction in any medium, provided the original work is properly cited. 